Waiting time between charging and discharging processes in molecular junctions

Daniel S. Kosov

Citation: The Journal of Chemical Physics 149, 164105 (2018); doi: 10.1063/1.5049770

View online: https://doi.org/10.1063/1.5049770

View Table of Contents: http://aip.scitation.org/toc/jcp/149/16

Published by the American Institute of Physics 


\title{
Waiting time between charging and discharging processes in molecular junctions
}

\author{
Daniel S. Kosov \\ College of Science and Engineering, James Cook University, Townsville, QLD 4811, Australia
}

(Received 25 July 2018; accepted 10 October 2018; published online 25 October 2018)

\begin{abstract}
When electric current flows through a molecular junction, the molecule constantly charges and discharges by tunneling electrons. These charging and discharging events occur at specific but random times and are separated by stochastic time intervals. These time intervals can be associated with the dwelling time for a charge (electron or hole) to reside on the molecule. In this paper, the statistical properties of these time intervals are studied and a general formula for their distribution is derived. The theory is based on the Markovian master equation which takes into account transitions between the vibrational states of charged and neutral molecules in the junction. Two quantum jump operators are identified from the Liouvillian of the master equation - one corresponds to charging of the molecule and the other discharges the molecule back to the neutral state. The quantum jump operators define the conditional probability that given that the molecule was charged by a tunneling electron at time $t$, the molecule becomes neutral at a later time $t+\tau$ discharging the electron to the drain electrode. Statistical properties of these time intervals $\tau$ are studied with the use of this distribution. Published by AIP Publishing. https://doi.org/10.1063/1.5049770
\end{abstract}

\section{INTRODUCTION}

Single-molecule electronics is the active field of research in chemical physics which has recently seen many advances. ${ }^{1,2}$ Molecular electronics has been promoted for decades as something to replace silicon based electronics (the dreams that never really came through), but rather it has successfully grown into an integral part of modern chemical physics that gives unique and otherwise unavailable opportunities to study the fundamental issues of quantum mechanics and nonequilibrium statistical physics of individual electron transfer events. $^{2-5}$

One of the main feature that distances molecular electronics from other nanoscale electron transport systems is the structural "softness" and as a consequence the possibility to observe current-induced "chemistry." ${ }^{-19}$ The electric current is an average quantity-it tells us how much energy is dissipated per unit time in the molecule, but current-triggered reactions do not only depend on the power pumped into the molecule but also on the dwelling time allowed for extra electrons (or holes) to reside on the molecular bridge (if the molecule remains charged for a considerable time when the electric field between electrodes has a chance to produce a significant deformation of the molecular geometry).

Electric current is a series of single electron quantum tunneling events separated by random time intervals, which means that the molecular bridge undergoes the continuous sequence of charging and discharging events also separated by random time intervals. This paper focuses on the statistical properties of these time intervals and addresses the following questions. How long does the fluctuating charge stay on the molecule when the current flows through it? What is the distribution of these times and what are the statistical properties of this distribution? How do the vibrational dynamics and the coupling between electronic and vibrational degrees of freedom influence the distribution of the charging times?

This work is based on the ideas of the waiting time distribution (WTD) - the theoretical approach to study statistics of individual electron tunneling events in nanoscale systems..$^{20,21}$ WTD is an extension of widely used in quantum transport methods of full counting statistics. $22-32$ WTD has recently gained a significant popularity in quantum transport research due to its intuitively clear interpretation and flexibility in the design of various extensions for a wide range of statistical applications. ${ }^{33-45}$ Traditional WTD is a conditional probability distribution to observe electron transfer event in the detector electrode at time $t+\tau$ given that an electron transfer was detected in the same electrode at earlier time $t$. WTDs are measured experimentally using time-resolved charge detection techniques for single-electron tunneling. ${ }^{46}$ The charge detection is usually implemented by monitoring changes in the electric current in auxiliary quantum point contact capacitively coupled to the main system. ${ }^{46-50}$ Most experiments have been conducted at ultra-low temperatures (from $\mathrm{mK}$ to several $\mathrm{K}$ ), although room temperature measurements were reported for a carbon nanotube by monitoring the optical blinking of semiconductor nanocrystals which is induced by the charging/discharging events in the nanonotube. ${ }^{51}$ The main limitation of all single-electron counting experimental methods is the restriction of counting no more than of approximately $10^{3}$ electrons per second (that is very low electric current). The interesting hybrid approach was proposed to extract WTD directly from low-order correlation experimental measurements via theoretical post-processing using continuous matrix product state tomography. ${ }^{52}$ 
In our recent studies, ${ }^{38,45}$ we used WTD to analyze the electron transport through molecular junctions with electronvibration coupling. In this paper, the WTD notion is adopted to study different kinds of probability distribution which is related to the temporal fluctuations of the molecular charging state rather than electric current, namely, we will define and explore the conditional probability distribution that given that the molecule was charged by the tunneling electron at time $t$, the molecule becomes neutral at a later time $t+\tau$ discharging an electron to the drain electrode. This type of WTD follows the same philosophy as tunneling and residence time distributions explored in papers. ${ }^{42,43}$ This distribution will be used in our paper to study statistical properties of temporal fluctuations of molecular charging states in a current-carrying junction with electron-vibration interaction.

The paper is organised as follows. Section II describes the master equations, defines charging and discharging quantum jump operators, and gives the derivation of main equations for WTDs. In Sec. III, we present analytical and numerical study of statistical properties of the waiting times between charging and discharging events in a molecular junction with electronvibration interaction. Section IV summarises the main results of the paper.

We use natural units for quantum transport throughout the paper, $\hbar=k_{B}=e=1$.

\section{THEORY}

To have a specific model, let us suppose that when an electron is transferred to the molecule from the electrodes, the molecule becomes negatively charged, and when this electron leaves, the molecule comes back to the neutral state. This scenario corresponds to the case of electron transport through a single resonant level above the equilibrium Fermi energy of the electrodes. The opposite case of a molecular bridge acquiring a positive charge (transport through a resonant level which is below the Fermi energy) can be considered likewise and we will simply give the final expression for this process for the comparison in the end of this section.

We begin with the following intuitively obvious master equation:

$$
\begin{aligned}
& \dot{P}_{0 q}(t)=\sum_{\alpha q^{\prime}} \Gamma_{0 q, 1 q^{\prime}}^{\alpha} P_{1 q^{\prime}}(t)-\Gamma_{1 q^{\prime}, 0 q}^{\alpha} P_{0 q}(t), \\
& \dot{P}_{1 q}(t)=\sum_{\alpha q^{\prime}} \Gamma_{1 q, 0 q^{\prime}}^{\alpha} P_{0 q^{\prime}}(t)-\Gamma_{0 q^{\prime}, 1 q}^{\alpha} P_{1 q}(t),
\end{aligned}
$$

where $P_{0 q}(t)$ is the probability that the molecule is neutral and occupied by $q$ vibrational quanta at time $t, P_{1 q}(t)$ is the probability that the molecule is charged and occupied by $q$ vibrational quanta at time $t$. The transition rates and the model are explained in Fig. 1. The rate $\Gamma_{0 q^{\prime}, 1 q}^{\alpha}$ describes the transition from the charged state and $q$ vibrations to the electronically neutral state with $q^{\prime}$ vibrations by the electron transfer from the molecule to $\alpha=S, D$ electrode and the rate $\Gamma_{1 q^{\prime}, 0 q}^{\alpha}$ describes the transition to the charged state from the originally neutral molecule by electron transfer from $\alpha$ electrode simultaneously changing the vibrational state from $q$ to $q^{\prime}$.

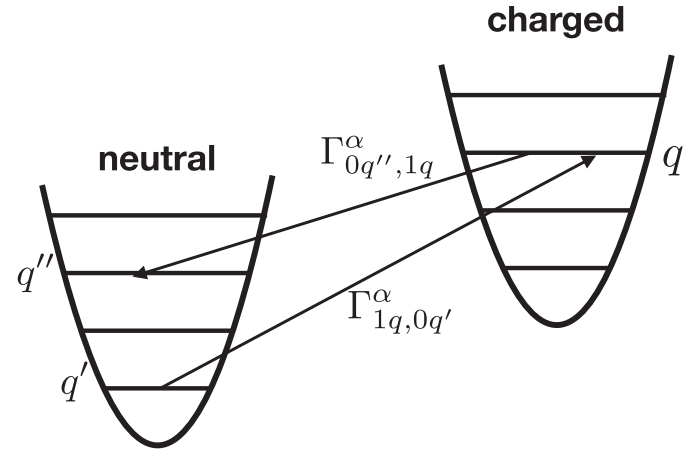

FIG. 1. Sketch of the model. $\Gamma_{m q, n q^{\prime}}^{\alpha}$ are the rates for the molecular charging or discharging processes by the transition of electron to/from source $\alpha=S$ and drain $\alpha=D$ electrodes by changing the molecular electronic state from $n$ to $m$ and simultaneously changing the molecular vibrational state from $q^{\prime}$ to $q$.

We will work with the probability vector ordered such that the probabilities to observe the molecule in neutral and charged states are grouped in pairs of equal number of the vibrational quanta:

$$
\mathbf{P}(t)=\left[\begin{array}{c}
P_{00}(t) \\
P_{10}(t) \\
P_{01}(t) \\
P_{11}(t) \\
\vdots \\
P_{0 N}(t) \\
P_{1 N}(t)
\end{array}\right],
$$

where $N$ is the total number of vibrational quanta included into the calculations. It is useful for our derivations to define the identity vector of length $2 \mathrm{~N}$

$$
\mathbf{I}=\left[\begin{array}{c}
1 \\
1 \\
1 \\
1 \\
\vdots \\
1 \\
1
\end{array}\right] .
$$

The normalisation of the probability is given by the scalar product

$$
(\mathbf{I}, \mathbf{P}(t))=1 .
$$

Using $\mathbf{P}(t)$, we write the master equation (1) and (2) in a matrix form

$$
\dot{\mathbf{P}}(t)=\mathcal{L} \mathbf{P}(t),
$$

where $\mathcal{L}$ is the Liouvillian operator. From the Liouvillian operator, we can identify two quantum jump operators corresponding to the processes of molecular charging and discharging by tunneling electrons. These quantum jump operators are $2 N \times 2 N$ matrices which are determined by considering their actions on the probability vector. For our discussion of charging and discharging processes, it is sufficient to define the two jump operators: the jump operator $J_{d}$ for transferring an electron from the molecule to the drain electrode (discharging in our case) 


$$
\left(J_{d} \mathbf{P}(t)\right)_{n q}=\delta_{n 0} \sum_{q^{\prime}} \Gamma_{0 q, 1 q^{\prime}}^{D} P_{1 q^{\prime}}(t)
$$

and the operator $J_{c}$ which describes electron transfer from the source electrode to the molecule (charging)

$$
\left(J_{c} \mathbf{P}(t)\right)_{n q}=\delta_{n 1} \sum_{q^{\prime}} \Gamma_{1 q, 0 q^{\prime}}^{S} P_{0 q^{\prime}}(t) .
$$

Each quantum jump is associated with the corresponding quantum measurement operator. If $\mathbf{P}(t)$ is the probability vector before the measurement, then after the quantum jump detection it collapses to the vector ${ }^{53}$

$$
M_{c / d} \mathbf{P}(t)=\frac{J_{c / d} \mathbf{P}(t)}{\left(\mathbf{I}, J_{c / d} \mathbf{P}(t)\right)},
$$

where $M_{c / d}$ is the quantum measurement operator related to the jump $J_{c / d}$.

We first extract from the total Liouvillian the part which generates the evolution without electron transfer from the molecule to the drain electrode

$$
\mathcal{L}_{0}=\mathcal{L}-J_{d}
$$

and re-write master equation (6) as

$$
\dot{\mathbf{P}}(t)=\left(\mathcal{L}_{0}+J_{d}\right) \mathbf{P}(t) .
$$

Next, (11) is converted to the integral equation

$$
\mathbf{P}(t)=e^{\mathcal{L}_{0} t} \mathbf{P}(0)+\int_{0}^{t} d t_{1} e^{\mathcal{L}_{0}\left(t-t_{1}\right)} J_{d} \mathbf{P}\left(t_{1}\right),
$$

which then is resolved as a series

$$
\mathbf{P}(t)=e^{\mathcal{L}_{0} t} \mathbf{P}(0)+\int_{0}^{t} d t_{1} e^{\mathcal{L}_{0}\left(t-t_{1}\right)} J_{d} e^{\mathcal{L}_{0} t_{1}} \mathbf{P}(0)+\cdots .
$$

We truncate this series at the first integral and that is sufficient to define the required time distributions. The next order terms in this expansion can be considered to define higher order probability distributions which describe correlations between two and more waiting times; for detailed discussion, we refer to Appendix A in Ref. 45.

The initial probability vector is chosen as

$$
\mathbf{P}(0)=M_{c} \mathbf{P},
$$

where $\mathbf{P}$ describes the non-equilibrium steady state. This choice of the initial state implies that the charging of the molecule by an electron transfer from the source electrode is detected at time $t=0$ in the steady state regime and then we start to monitor the system for discharging events

$$
\mathbf{P}(t)=e^{\mathcal{L}_{0} t} M_{c} \mathbf{P}+\int_{0}^{t} d t_{1} e^{\mathcal{L}_{0}\left(t-t_{1}\right)} J_{d} e^{\mathcal{L}_{0} t_{1}} M_{c} \mathbf{P} .
$$

Using electron detection operator (9), we rewrite (14) in a form which explains the probabilistic meanings of the integral term and, consequently, enables extraction of an expression for the probability distribution of the time delay between charging and discharging events
$\mathbf{P}(t)=e^{\mathcal{L}_{0} t} M_{c} \mathbf{P}+\int_{0}^{t} d t_{1} \frac{\left(\mathbf{I}, J_{d} e^{\mathcal{L}_{0} t_{1}} J_{c} \mathbf{P}\right)}{\left(\mathbf{I}, J_{c} \mathbf{P}\right)} e^{\mathcal{L}_{0}\left(t-t_{1}\right)} M_{d} e^{\mathcal{L}_{0} t_{1}} M_{c} \mathbf{P}$.

Let us now interpret (15). The first term, $e^{\mathcal{L}_{0} t} M_{c} \mathbf{P}$, is the contribution to the probability vector from all measurements where no electron transfer from the molecule to the drain electrode occurs up to time $t$ after the initial molecular charging at time $t=0$. The physical meaning of the integral term in (15) is deduced using the following arguments..$^{454-56}$ The molecule is charged by transferring an electron into the molecule from the source electrode at time $t=0\left(\operatorname{term} M_{c} \mathbf{P}\right)$, then no detection of an electron transfer to the drain electrode occurs up to time $t_{1}$ ("idle" evolution operator $e^{\mathcal{L}_{0} t_{1}}$ ), then the detection of the discharging of the molecule by transferring an electron to the drain electrode is observed at time $t_{1}$ (quantum measurement operator $M_{d}$ ), and finally the system "idle" without electron transfer up to time $t$. Therefore, the pre-factor $\left(\mathbf{I}, J_{d} e^{\mathcal{L}_{0} t_{1}} J_{c} \mathbf{P}\right) /\left(\mathbf{I}, J_{c} \mathbf{P}\right)$ should be understood as the probability of observing this process.

The main result of this section is summarized in the equation below. The distribution of waiting times between charging and discharging events in the molecular junction is

$$
w(t)=\frac{\left(\mathbf{I}, J_{d} e^{\mathcal{L}_{0} t} J_{c} \mathbf{P}\right)}{\left(\mathbf{I}, J_{c} \mathbf{P}\right)} .
$$

The derivations for fluctuating positive charge (transport through highest occupied molecular orbital resonant level) can be performed along exactly the same lines and give

$$
w(t)=\frac{\left(\mathbf{I}, J_{c} e^{\mathcal{L}_{0} t} J_{d} \mathbf{P}\right)}{\left(\mathbf{I}, J_{d} \mathbf{P}\right)} .
$$

These distributions are conceptually similar to tunneling and residence time distributions proposed for electron transport through a single resonant level, ${ }^{42,43}$ though we use different definition of the "idle" Liouvillian here. It is instructive to contrast definitions (16) and (17) with standard WTD used in electron transport theory which is a conditional probability to observe the electron transfer in the detector electrode given that an electron was detected in the same detector electrode at earlier time $\left(\mathbf{I}, J_{d} e^{\mathcal{L}_{0} t} J_{d} \mathbf{P}\right) /\left(\mathbf{I}, J_{d} \mathbf{P}\right) .{ }^{20}$

We would like to make an important note on the range of validity of the developed theory. The expressions for WTD derived here technically work for bidirectional and unidirectional electron transport. However, the rigorous interpretation of (16) and (17) as conditional distributions of delay times between charging and discharging events is possible only in the absence of the electron back-tunneling against the average current flow (tunneling from the drain electrode to the molecule or from the molecule to the source electrode). If the back-tunneling is not suppressed by the choice of the applied voltage and other parameters of the model, the charging events from the reverse tunneling electrons become physically present but they are concealed inside the idle time-evolution operator $e^{\mathcal{L}_{0} t}$ and are not being explicitly monitored in (16) and (17). For the case of bidirectional current, one should think about WTDs given by (16) and (17) as conditional distribution of waiting 
time between events of electron tunneling to the drain electrode and electron tunneling from the source electrode (with back-tunneling charging and discharging events covert in the waiting time intervals).

\section{MODEL CALCULATIONS}

Our study of statistical properties of time delays separating charging and discharging events will be based on the following idealised model. The molecule is attached to two macroscopic leads (source and drain) held at different chemical potentials and is represented by a single molecular orbital linearly coupled to local vibration. The total Hamiltonian of molecular junction is

$$
H=H_{\text {molecule }}+H_{\text {electrodes }}+H_{T} .
$$

The molecular Hamiltonian is

$$
H_{\text {molecule }}=\epsilon_{0} a^{\dagger} a+\omega b^{\dagger} b+\lambda a^{\dagger} a\left(b^{\dagger}+b\right),
$$

where $\epsilon_{0}$ is the energy or the molecular orbital, $\omega$ is the vibrational frequency, and $\lambda$ is the strength of electron-vibration coupling. $a^{\dagger}(a)$ creates (annihilates) an electron on molecular orbital, and $b^{+}(b)$ is a bosonic creation (annihilation) operator for the molecular vibration.

Electrodes consist of noninteracting electrons

$$
H_{\text {electrodes }}=\sum_{k \alpha} \epsilon_{k \alpha} a_{k \alpha}^{\dagger} a_{k \alpha},
$$

where $a_{k \alpha}^{\dagger}$ creates an electron in the single-particle state $k$ of $\alpha$ electrode and $a_{k \alpha}$ is the corresponding electron annihilation operator. The electron tunneling is described by

$$
H_{T}=\sum_{k \alpha}\left(t_{k \alpha} a_{k \alpha}^{\dagger} a+h . c\right),
$$

where $t_{k \alpha}$ are the tunneling amplitudes.

The Lang-Firsov unitary rotation (polaron transformation) of molecular operators ${ }^{57}$ is used to remove electronvibration coupling from the molecular Hamiltonian

$$
a=\tilde{a} e^{v\left(\tilde{b}^{\dagger}-\tilde{b}\right)}, \quad b=\tilde{b}+v \tilde{a}^{\dagger} \tilde{a},
$$

where $\tilde{a}^{\dagger}(\tilde{a})$ and $\tilde{b}^{\dagger}(\tilde{b})$ are new creation (annihilation) operators for the molecular electron and vibration. The molecular Hamiltonian becomes

$$
H_{\text {molecule }}=\epsilon \tilde{a}^{\dagger} \tilde{a}+\omega \tilde{b}^{\dagger} \tilde{b}
$$

where the molecular orbital energy $\epsilon$ includes the polaron shift $\epsilon=\epsilon_{0}-\lambda^{2} / \omega$. The Hamiltonian for the electrodes is invariant under Lang-Firsov rotation and the tunneling interaction becomes

$$
H_{T}=\sum_{k \alpha}\left(t_{k \alpha} e^{-\frac{\lambda}{\omega}\left(\tilde{b}^{\dagger}-\tilde{b}\right)} a_{k \alpha}^{\dagger} \widetilde{a}+h . c\right) .
$$

Now, after Lang-Firsov transformation, the molecular Hamiltonian (23) is diagonal. Next, the application of the sequence of approximations, the Born approximation (keeping terms up to second order in $H_{T}$ in the Liouville equation for the reduced density matrix), the Markov approximation (assumption that the correlation functions of the electrodes decay on a time scale much faster than tunneling events), and the secular approximations (amounts to neglect coherences between charge states of the molecule), leads to the master equation (1) and (2) with the following transition rates: ${ }^{58}$

$$
\Gamma_{0 q^{\prime}, 1 q}^{\alpha}=\gamma^{\alpha}\left|X_{q^{\prime} q}\right|^{2}\left(1-f_{\alpha}\left[\epsilon-\omega\left(q^{\prime}-q\right)\right]\right)
$$

and

$$
\Gamma_{1 q^{\prime}, 0 q}^{\alpha}=\gamma^{\alpha}\left|X_{q^{\prime} q}\right|^{2} f_{\alpha}\left[\epsilon+\omega\left(q^{\prime}-q\right)\right] .
$$

The rates depend on Fermi-Dirac occupation numbers for the electrode states $f_{\alpha}$, Franck-Condon factor $X_{q q^{\prime}}$, and electronic level broadening function $\gamma^{\alpha}$. The Franck-Condon factor

$$
X_{q q^{\prime}}=\left\langle q\left|e^{-\lambda / \omega\left(b^{\dagger}-b\right)}\right| q^{\prime}\right\rangle
$$

is determined by the strength of the electron-vibration coupling $\lambda$.

Master equations (1) and (2) describes non-equilibrium dynamics of the molecular vibrations and this fully nonequilibrium case will shortly be considered numerically. First, we take the limit where the vibration is maintained in thermodynamic equilibrium at some temperature $T$; it enables us to obtain analytical expressions for the probability distributions. This limit physically means that the molecular vibration is attached to its own bath, which can be, for example, a solvent around the molecular junction or surface phonons in metal electrodes.

To implement this limit, we use the following separable ansatz for the probabilities ${ }^{58}$

$$
P_{n q}(t)=P_{n}(t) \frac{e^{-q \omega / T}}{1-e^{-\omega / T}},
$$

which assumes that the vibration maintains the equilibrium distribution at all time. The master equation (1) and (2) is reduced to the evolution equation for the probabilities to observe the molecule in neutral and charged states, $P_{0}$ and $P_{1}$, respectively,

$$
\frac{d}{d t}\left[\begin{array}{l}
P_{0} \\
P_{1}
\end{array}\right]=\left[\begin{array}{cc}
-\Gamma_{10} & \Gamma_{01} \\
\Gamma_{10} & -\Gamma_{01}
\end{array}\right]\left[\begin{array}{l}
P_{0} \\
P_{1}
\end{array}\right],
$$

where the vibration averaged rates are defined as

$$
\Gamma_{m n}^{\alpha}=\sum_{q q^{\prime}} \Gamma_{m q, n q^{\prime}}^{\alpha} \frac{e^{-q^{\prime} \omega / T}}{1-e^{-\omega / T}}
$$

and the total rates include contributions from the source and drain electrodes $\Gamma_{m n}=\sum_{\alpha} \Gamma_{m n}^{\alpha}$. We write discharging and charging quantum jump operators in a matrix form and also as a dyadic product of two vectors

$$
\begin{aligned}
& J_{d}=\left[\begin{array}{cc}
0 & \Gamma_{01}^{D} \\
0 & 0
\end{array}\right]=\Gamma_{01}^{D}\left[\begin{array}{l}
1 \\
0
\end{array}\right]\left[\begin{array}{ll}
0 & 1
\end{array}\right], \\
& J_{c}=\left[\begin{array}{cc}
0 & 0 \\
\Gamma_{10}^{S} & 0
\end{array}\right]=\Gamma_{10}^{S}\left[\begin{array}{l}
0 \\
1
\end{array}\right]\left[\begin{array}{ll}
1 & 0
\end{array}\right] .
\end{aligned}
$$

Next, straightforward vector algebra brings the WTD between charging and discharging events to

$$
w_{e q}(\tau)=\Gamma_{01}^{D}\left[\begin{array}{ll}
0 & 1
\end{array}\right] e^{\mathcal{L}_{0} \tau}\left[\begin{array}{l}
0 \\
1
\end{array}\right],
$$


where

$$
\mathcal{L}_{0}=\mathcal{L}-J_{d}=\left[\begin{array}{cc}
-\Gamma_{10} & \Gamma_{01}^{S} \\
\Gamma_{10} & -\Gamma_{01}
\end{array}\right] .
$$

Here the "eq" subscript in the time distribution indicates the equilibrium molecular vibration (and, of course, electrons are still in non-equilibrium). This expression can be further evaluated and brought to the following analytic form:

$$
\begin{aligned}
w_{e q}(\tau)= & \frac{\Gamma_{01}^{D}}{2 Z}\left\{\left(Z+\Gamma_{01}-\Gamma_{10}\right) e^{-\left(Z+\Gamma_{01}+\Gamma_{10}\right) t / 2}\right. \\
& \left.+\left(Z+\Gamma_{10}-\Gamma_{01}\right) e^{-\left(\Gamma_{01}+\Gamma_{10}-Z\right) t / 2}\right\},
\end{aligned}
$$

where

$$
Z=\sqrt{\left(\Gamma_{01}-\Gamma_{10}\right)^{2}+4 \Gamma_{10} \Gamma_{01}^{S}} .
$$

The bi-exponential dependence of the distribution function is a consequence of rare extreme events, electron tunneling against the current flow from the molecule to the source electrode or from the drain electrode back to the molecule. Suppressing the back-scattering electron transfer,

$$
\Gamma_{10}^{D} \rightarrow 0, \quad \Gamma_{01}^{S} \rightarrow 0
$$

yields single-exponent WTD

$$
w_{e q}(\tau)=\Gamma_{01}^{D} e^{-\Gamma_{01}^{D} \tau} .
$$

Figure 2 shows the WTDs for between molecular charging and discharging processes computed for different values of the electron-vibration coupling strength. WTDs for equilibrated vibration are calculated using (34) and fully nonequilibrium WTDs are computed numerically via (16). All distributions attain their maximum values at $\tau=0$; therefore the mode waiting times between charging and discharging

(a)

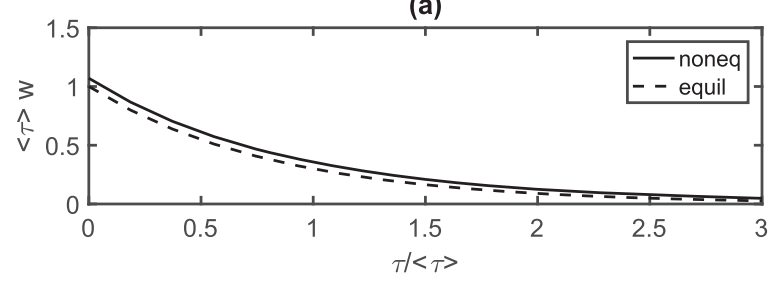

(b)

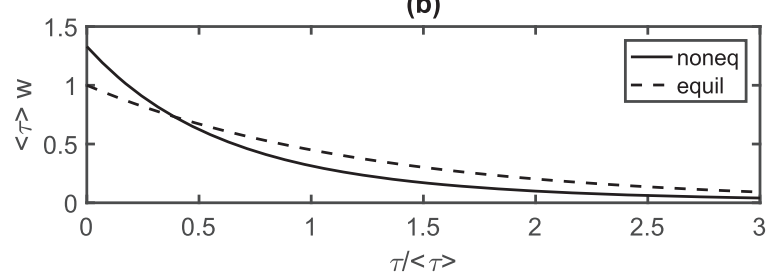

(c)

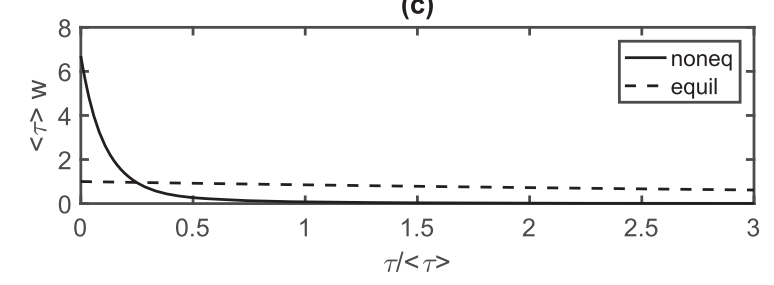

FIG. 2. WTD between molecular charging and discharging events computed for different values of the electron-vibrational coupling strength: (a) $\lambda=1$, (b) $\lambda=2$, and (c) $\lambda=3$. Parameters used in calculations: $\omega=1, \gamma_{S}=\gamma_{D}$ $=0.01, T=0.05, \epsilon=0.1$, and $V_{s d}=4$. All energy values are given in units of $\omega$. process are always zero. It indicates that the short time behavior of time delays between charging and discharging events is dominated by the Poisson point process noise. It is not clear at the moment if this short time behavior is merely a result of approximations used to derive the underlying master equation (Markov and secular approximations, in particular). The electron-vibrational interaction has the opposite effect on the behavior of time distributions for equilibrium and non-equilibrium molecular vibrations. The increasing strength of electron-vibrational interaction significantly squeezes the non-equilibrium time distribution, but the equilibrium one is just sightly stretched to the longer than average waiting times. These different behaviors can be understood based on the following considerations. In the equilibrium regime, the molecular vibrational state is forced to be populated to a given temperature irrespective to electronic degrees of freedom and, as a result, the time distribution function (when scaled by its average waiting time $\langle\tau\rangle$ ) shows only small dependence on the strength of the electron-vibration coupling. This means that in the case of forcefully equilibrated molecular vibrations, the strength of the electron-vibration coupling affects mostly the average value leaving the other WTD parameters intact. Contrarily, in the non-equilibrium case, the different numbers of vibrational quanta can be dynamically excited and de-excited by tunneling electrons and, therefore, the electron-vibration interaction plays a critical role for these processes.

Figure 3 shows the average time delay between molecular charging and discharging events as a function of the voltage bias for different strengths of the electron-vibration coupling. The steps in the average time is related to the resonant excitations of the vibration states by electric currents which occur when the voltage passes through an integer multiple of the vibrational energy. The temperature effects smooth the edges

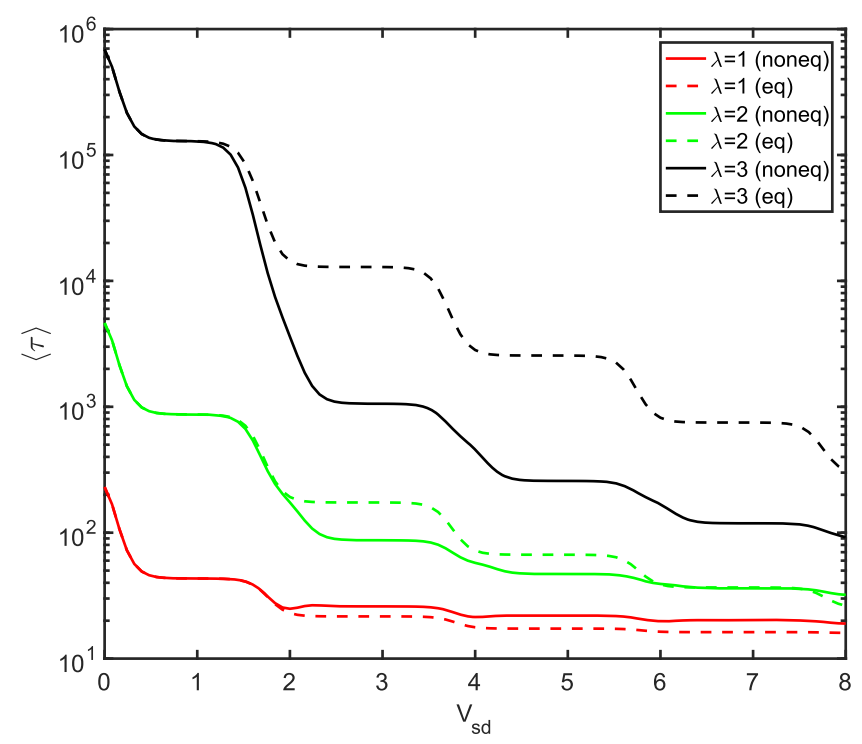

FIG. 3. Average waiting time between charging and discharging processes as a function of applied voltage $V_{s d}$ computed for different values of electronvibrational coupling $\lambda$. Parameters used in calculations (all energy values are given in units of $\omega$ ): $\omega=1, \gamma_{S}=\gamma_{D}=0.01, T=0.05, \epsilon=0.1$. The voltage bias $V_{s d}$ is given in $\omega$ and the time is measured in periods of the molecular vibration $2 \pi / \omega$. 
of these steps. This behavior reflects the staircase dependence of the electric current on the voltage ${ }^{58}$ the sharp increase of charging state lifetimes in the regime of strong coupling and low voltage corresponds to the Franck-Condon blockade suppression of the electric current. ${ }^{59}$

Figure 4 shows the relative standard deviation (RSD)

$$
\operatorname{RSD}=\frac{\sqrt{\left\langle\tau^{2}\right\rangle-\langle\tau\rangle^{2}}}{\langle\tau\rangle}
$$

computed for different strengths of electron-vibrational coupling as a function of applied voltage bias. Notice that mathematically, a single exponential arbitrary distribution has always $\operatorname{RSD}=1$, irrespective of the parameters. At small voltages, the RSDs are greater than 1 for both equilibrium and non-equilibrium vibrational dynamics indicating a multiexponential character of the WTDs-this is due to the admixture of back-scattering electron transfers from the molecule back to the source electrode or from the drain electrode to the molecule. When the backscattering processes are suppressed by the voltage bias $\left(V_{s d} \gtrsim 0.5\right)$, the RSD becomes exactly 1 for the equilibrium vibrations which means that $w_{e q}(\tau)$ is reduced to a single exponential form (37). The WTD for non-equilibrium vibrations also becomes single exponential in the voltage range $0.5 \lesssim V_{s d} \lesssim 1.5$ since here the backscattering processes are already suppressed by the voltage bias but inelastic vibrational channels for electron transport have not been opened yet. The further increase of voltage above the excitation threshold for inelastic channels leads to the increased RSD, which means that the non-equilibrium WTD becomes a multi-exponential (i.e., multichannel) distribution.

(a)

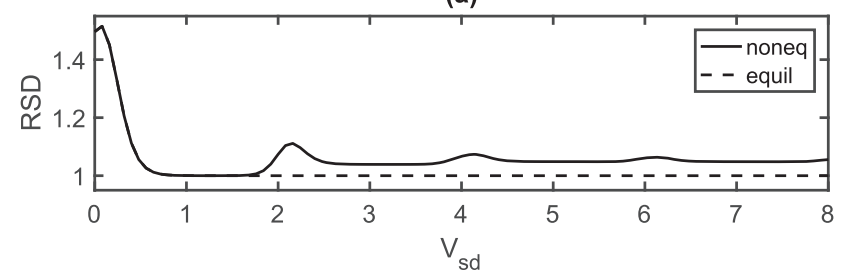

(b)

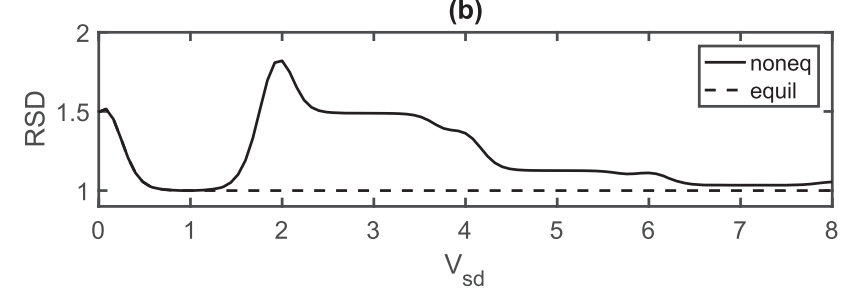

(c)

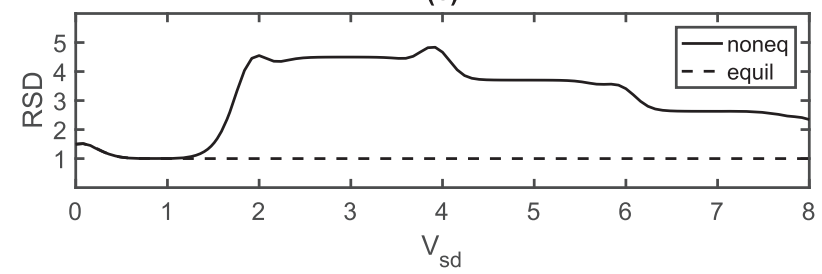

FIG. 4. Relative standard deviation (38) as a function of applied voltage $V_{s d}$ computed for different values of the electron-vibrational coupling strength: (a) $\lambda=1$, (b) $\lambda=2$, and (c) $\lambda=3$. Parameters used in calculations (all energy values are given in units of $\omega): \omega=1, \gamma_{S}=\gamma_{D}=0.01, T=0.05, \epsilon=0.1$. The voltage bias $V_{s d}$ is given in $\omega$.
The numerical calculations elucidate the origin of small spikes in the RSD voltage dependence seen in Fig. 4 for the non-equilibrium vibrations. These spikes occur only at the opening of new vibrational transport channels and they are due to electron backscattering events governed by the rates $\Gamma_{1 q, 0 q^{\prime}}^{D}$ and $\Gamma_{0 q, 1 q^{\prime}}^{S}$ with $q, q^{\prime}>0$. If these rates are set to zero, the RSD spikes vanish. The parallel can be drawn with the well-known phenomena in electron current noise-the noise is small when the single channel dominates the transport and grows once the electron transport is distributed along several channels.

\section{CONCLUSIONS}

We have developed a theoretical approach to compute statistical distributions of waiting times between charging and discharging process in a molecular junction with electronvibration interactions. The approach is based on the use of the Markovian master equation with the exact treatment of electron-vibration interactions to describe electron transport through a molecular junction. Two quantum jump operators responsible for molecular bridge charging and discharging were extracted from the Liouvillian of the master equation. These jump operators were used to develop WTD for time delays between charging and discharging events. The statistics of these events were studied analytically and numerically for a model molecular junction described by the Holstein Hamiltonian.

The main observations are as follows:

- For the case of equilibrium vibrations, the distribution of waiting times between molecular charging and discharging processes is bi-exponential-the dominant exponent represents the electron transport from the molecule to the drain electrode, while the other exponent reflects the presence of the rare (at high voltage) electron tunneling events where an electron is moving against the average electric current flow.

- The WTDs between charging and discharging events have distinct dependence of the strength of electronvibration coupling. For weak electron-vibration coupling $(\lambda=1)$, there is not much difference between WTDs for equilibrium and non-equilibrium vibrations, whereas as the strength of electron-vibration coupling grows $(\lambda=2,3)$, the non-equilibrium WTD narrows down to its mode time $(\tau=0)$, but equilibrium WTD spreads to the much longer than its average waiting times. Once scaled by their average waiting times, the equilibrium WTD shows much less dependence on $\lambda$ than its non-equilibrium counterpart.

- Analysis of the WTDs using RSD (the noise-to-signal ratio for measuring the average waiting time between charging and discharging processes) shows that dynamical openings of elastic and inelastic vibrational transport channels by the increasing voltage bias lead to significant increase of the RSD $(>1)$ for non-equilibrium vibrational dynamics indicating the multi-exponential nature of non-equilibrium WTD. 


\section{ACKNOWLEDGMENTS}

The author thanks Samuel Rudge for many valuable and stimulating discussions.

${ }^{1}$ M. Thoss and F. Evers, J. Chem. Phys. 148, 030901 (2018).

${ }^{2}$ D. Xiang, X. Wang, C. Jia, T. Lee, and X. Guo, Chem. Rev. 116, 4318 (2016).

${ }^{3}$ A. Nitzan and M. A. Ratner, Science 300, 1384 (2003).

${ }^{4}$ M. Galperin, M. A. Ratner, and A. Nitzan, J. Phys.: Condens. Matter 19, 103201 (2007).

${ }^{5}$ R. Nichols, W. Haiss, S. J. Higgins, E. Leary, S. Martin, and D. Bethell, Phys. Chem. Chem. Phys. 12, 2801 (2010).

${ }^{6}$ B. C. Stipe, M. A. Rezaei, W. Ho, S. Gao, M. Persson, and B. I. Lundqvist, Phys. Rev. Lett. 78, 4410 (1997).

${ }^{7}$ L. J. Lauhon and W. Ho, Phys. Rev. Lett. 84, 1527 (2000).

${ }^{8}$ H. J. Lee and W. Ho, Science 286, 1719 (1999).

${ }^{9}$ S.-W. Hla, L. Bartels, G. Meyer, and K.-H. Rieder, Phys. Rev. Lett. 85, 2777 (2000).

${ }^{10}$ J. Repp, G. Meyer, S. Paavilainen, F. E. Olsson, and M. Persson, Science 312, 1196 (2006).

${ }^{11}$ A. A. Dzhioev and D. S. Kosov, J. Chem. Phys. 135, 074701 (2011).

${ }^{12}$ M. Thomas, T. Karzig, S. V. Kusminskiy, G. Zaránd, and F. von Oppen, Phys. Rev. B 86, 195419 (2012).

${ }^{13}$ J.-T. Lu, M. Brandbyge, and P. Hedegard, Nano Lett. 10, 1657 (2010).

${ }^{14}$ A. A. Dzhioev, D. S. Kosov, and F. von Oppen, J. Chem. Phys. 138, 134103 (2013).

${ }^{15}$ A. Erpenbeck, C. Schinabeck, U. Peskin, and M. Thoss, Phys. Rev. B 97, 235452 (2018).

${ }^{16} \mathrm{H}$. Li, T. A. Su, V. Zhang, M. L. Steigerwald, C. Nuckolls, and L. Venkataraman, J. Am. Chem. Soc. 137, 5028 (2015).

${ }^{17}$ A. C. Aragonés, N. L. Haworth, N. Darwish, S. Ciampi, N. J. Bloomfield, G. G. Wallace, I. Diez-Perez, and M. L. Coote, Nature 531, 88 (2016).

${ }^{18}$ I. V. Pobelov, K. P. Lauritzen, K. Yoshida, A. Jensen, G. Mészáros, K. W. Jacobsen, M. Strange, T. Wandlowski, and G. C. Solomon, Nat. Commun. 8, 15931 (2017).

${ }^{19}$ G. Foti and H. Vázquez, J. Phys. Chem. Lett. 9, 2791 (2018).

${ }^{20}$ T. Brandes, Ann. Phys. 17, 477 (2008).

${ }^{21}$ M. Albert, G. Haack, C. Flindt, and M. Büttiker, Phys. Rev. Lett. 108, 186806 (2012).

${ }^{22}$ T.-H. Park and M. Galperin, Phys. Rev. B 84, 205450 (2011).

${ }^{23}$ Y. Utsumi, O. Entin-Wohlman, A. Ueda, and A. Aharony, Phys. Rev. B 87, 115407 (2013).

${ }^{24}$ K. Kaasbjerg and W. Belzig, Phys. Rev. B 91, 235413 (2015).

${ }^{25}$ R. Avriller and A. Levy Yeyati, Phys. Rev. B 80, 041309 (2009).

${ }^{26}$ C. Schinabeck, R. Härtle, H. B. Weber, and M. Thoss, Phys. Rev. B 90, 075409 (2014).

${ }^{27}$ B. K. Agarwalla, J.-H. Jiang, and D. Segal, Phys. Rev. B 92, 245418 (2015).
${ }^{28}$ G. Schaller, T. Krause, T. Brandes, and M. Esposito, New J. Phys. 15, 033032 (2013).

${ }^{29}$ D. A. Bagrets and Y. V. Nazarov, Phys. Rev. B 67, 085316 (2003).

${ }^{30}$ Y. V. Nazarov and Y. M. Blanter, Quantum Transport: Introduction to Nanoscience (Cambridge University Press, 2009).

${ }^{31}$ S. T. Carr, D. A. Bagrets, and P. Schmitteckert, Phys. Rev. Lett. 107, 206801 (2011).

${ }^{32}$ M. Ridley, V. N. Singh, E. Gull, and G. Cohen, Phys. Rev. B 97, 115109 (2018).

${ }^{33}$ G.-M. Tang, F. Xu, and J. Wang, Phys. Rev. B 89, 205310 (2014).

${ }^{34}$ K. H. Thomas and C. Flindt, Phys. Rev. B 87, 121405 (2013).

${ }^{35}$ K. H. Thomas and C. Flindt, Phys. Rev. B 89, 245420 (2014).

${ }^{36}$ D. Dasenbrook, P. P. Hofer, and C. Flindt, Phys. Rev. B 91, 195420 (2015).

${ }^{37}$ E. Potanina and C. Flindt, Phys. Rev. B 96, 045420 (2017).

${ }^{38}$ D. S. Kosov, J. Chem. Phys. 146, 074102 (2017).

${ }^{39}$ R. Seoane Souto, R. Avriller, R. C. Monreal, A. Martín-Rodero, and A. Levy Yeyati, Phys. Rev. B 92, 125435 (2015).

${ }^{40}$ B. Sothmann, Phys. Rev. B 90, 155315 (2014).

${ }^{41}$ H. P. Goswami and U. Harbola, J. Chem. Phys. 142, 084106 (2015).

${ }^{42}$ S. L. Rudge and D. S. Kosov, J. Chem. Phys. 144, 124105 (2016).

${ }^{43}$ S. L. Rudge and D. S. Kosov, Phys. Rev. E 94, 042134 (2016).

${ }^{44}$ K. Ptaszyński, Phys. Rev. B 95, 045306 (2017).

${ }^{45}$ D. S. Kosov, J. Chem. Phys. 147, 104109 (2017).

${ }^{46}$ S. Gustavsson, R. Leturcq, M. Studer, I. Shorubalko, T. Ihn, K. Ensslin, D. C. Driscoll, and A. C. Gossard, Surf. Sci. Rep. 64, 191 (2009).

${ }^{47}$ W. Lu, Z. Ji, L. Pfeiffer, K. W. West, and A. J. Rimberg, Nature 423, 422 (2003).

${ }^{48}$ L. M. K. Vandersypen, J. M. Elzerman, R. N. Schouten, L. H. Willems van Beveren, R. Hanson, and L. P. Kouwenhoven, Appl. Phys. Lett. 85, 4394 (2004).

${ }^{49}$ N. Ubbelohde, C. Fricke, C. Flindt, F. Hohls, and R. J. Haug, Nat. Commun. 3, 612 (2012).

${ }^{50}$ S. Gustavsson, R. Leturcq, B. Simovič, R. Schleser, T. Ihn, P. Studerus, K. Ensslin, D. C. Driscoll, and A. C. Gossard, Phys. Rev. Lett. 96, 076605 (2006).

${ }^{51}$ E. Zbydniewska, A. Duzynska, M. Popoff, D. Hourlier, S. Lenfant, J. Judek, M. Zdrojek, and T. Mélin, Nano Lett. 15, 6349 (2015).

${ }^{52}$ G. Haack, A. Steffens, J. Eisert, and R. Hübener, New J. Phys. 17, 113024 (2015).

${ }^{53}$ H. P. Breuer and F. Petruccione, The Theory of Open Quantum Systems (Oxford University Press, Oxford, 2002).

${ }^{54}$ M. Srinivas and E. Davies, Opt. Acta: Int. J. Opt. 28, 981 (2010).

${ }^{55}$ P. Zoller, M. Marte, and D. Walls, Phys. Rev. A 35, 198 (1987).

${ }^{56}$ A. A. Budini, J. Phys. B: At., Mol. Opt. Phys. 43, 115501 (2010).

${ }^{57}$ I. G. Lang and Y. A. Firsov, Sov. J. Exp. Theor. Phys. 16, 1301 (1963).

${ }^{58}$ A. Mitra, I. Aleiner, and A. J. Millis, Phys. Rev. B 69, 245302 (2004).

${ }^{59}$ J. Koch and F. von Oppen, Phys. Rev. Lett. 94, 206804 (2005). 